\title{
The challenge of acute non-compressive transverse myelopathies
}

\author{
Ambar Chakravarty* \\ Department of Neurology, Vivekananda Institute of Medical Science, Calcutta, India \\ ${ }^{*}$ Correspondence: saschakra@yahoo.com
}

The term 'myelopathy' is used in this brief critical communication in preference to the commonly used term 'myelitis' to highlight that all cases of acute transverse myelopathies are not of inflammatory origin. Indeed metabolic and vascular myelopathies, at times, do pose serious diagnostic problems and need different therapeutic approaches.

The first question, when faced with a clinically obvious transverse myelopathy, is whether it is really a 'new' 'acute' onset disease or an 'acute' exacerbation of a preexisting unsuspected or unknown compressive or non-compressive myelopathic problem or perhaps a super addition of an acute non-compressive pathology on top of a mild pre-existing compressive problem. This issue needs to be settled at the very outset by careful history elicitation including family history and a very thorough neuroradiological assessment. It must be stressed that spinal MRI must involve the entire spine with contrast and not limited only to the clinically suggestive level, not only to exclude unsuspected compressive pathology elsewhere but also to gauge the full length of the detected spinal lesion and exclude multiplicity of similar lesions elsewhere in the spinal cord. Even after careful radiological study, it may be sometimes difficult to differentiate inflammatory lesions from intramedullary tumors, subtle extramedullary spinal cord compression with marked signal changes in the spinal cord at the same level or even extending above and below the level including hematoma and/or ischemic events. Imaging should preferably include the brain as well if an inflammatory process is suspected. Establishing the etiology must also include cerebrospinal fluid (CSF) analysis with immunological studies. Two recent reviews by Brinar et al. (2008) and Jacob and Weinshenker (2008) detail the differential diagnosis and of acute transverse myelopathies. The latter workers (Jacob and Weinshenker, 2008) have presented a very clinically oriented approach to finding the etiology based on anatomical localization of region of cord affected as revealed by thorough clinical and radiological means. They have classified all intrinsic cord syndromes as - complete, Brown-Séquard syndrome, anterior cord syndrome, posterior cord syndrome, central cord syndrome, conus medullaris syndrome, cauda equina syndrome, and tractopathies. This appears to be an excellent approach. These authors (Jacob and Weinshenker, 2008) have also provided a 'flow chart' approach to myelopathies which seems to be very useful for the practicing clinician.

Acute inflammatory myelopathies can not solely be due to established autoimmune central nervous system (CNS) demyelinating disorders of CNS like multiple sclerosis (MS), acute disseminated encephalomyelitis (ADEM) or neuromyelitis optica (NMO), but may also complicate several systemic autoimmune disorders including systemic lupus erythematosus (SLE), Sjogren's syndrome, antiphospholipid antibody syndrome, mixed connective tissue disease, systemic sclerosis, ankylosing spondylitis and sarcoidosis.

The talk of the day is of course the discovery of the Aquaporin4 antibody (NMO-IgG), a hallmark of the NMO spectrum disorders with long segment spinal cord signal changes with or without optic nerve involvement. NMO is now generally regarded as quite distinct from MS as the prognosis and radiology are somewhat different. However, the antibody may also be detected in some cases of classical MS and may be undetectable in as high as 30\% cases of classical NMO. NMO-IgG antibody is not simply a diagnostic marker but has a pathological bearing. This represents a unique dysfunction of water channels which has been linked to the spinal cord edema in NMO with intact blood brain barrier (no GAD enhancement) and this helps in differentiation from spinal lesion in ADEM (GAD enhancing). Earlier workers from Japan and India pointed out at the long segment spinal lesions (three segments or more) in Asian patients with MS. These studies were done before the publication of the revised McDonald (Polman et al., 2005) criteria for diagnosis of MS and before the availability of the NMO-IgG antibody test. It is essential to review such cases both in terms of the current diagnostic criteria of MS as well as presence or absence of the antibody in the CSF. The wider availability of the antibody test and at a lesser cost in developing Asian countries need not be overemphasized.

A major clinical problem arises in patients with clinical features of a transverse myelopathy associated with a normal spinal MRI. The first thing would be to go back on clinical details to make sure whether it was indeed a myelopathy and not an atypical presentation of a peripheral neuropathic lesion (like Guillain-Barré syndrome) or a rare presentation of a cerebral pathology. The second would be a careful review of the whole spine contrast enhanced MRI and if needed, a repeat MRI. Having done so, the CSF needs careful examination. This might show the presence of oligoclonal bands or a raised CSF protein content but no vigorous inflammatory response. These along with the lack of radiologically demonstrable demyelination, might suggest that parenchymatous neurons are the sites of pathologic involvement, analogous to the acute lesions of axonal polyneuropathy. Such cases would be variants of ADEM although similar cases had been described in patients with SLE and regarded as part of the NMO spectrum (Jacobi et al., 2006).

The final and essential challenge is assessment of risk of recurrence. Complete transverse myelitis patients in general are at low risk of future development of MS. However, they could have recurrences consistent with relapsing myelitis or NMO. Two immune markers that may predict recurrence are anti-Sjögren syndrome antibody (SS-A) and NMO-IgG. The latter had been extensively 
studied and is a must in all cases of transverse myelopathies. Patients with incomplete transverse myelitis, on the other hand can be considered to have clinically isolated forms of MS (CIS) and the risk of future development of MS would remain a worry. When associated with brain lesions the risk is high (over $80 \%$ ), but with no associated brain lesions the risk is relatively low (less than 20\%). The diagnosis of acute infective myelitis, radiation myelopathies and paraneoplastic acute myelopathies, are relatively simple. Diagnosing the latter however, demands strong clinical suspicion, thorough clinical and radiological search for hidden neoplasia coupled with testing for a wide variety of paraneoplastic antibodies in cases where the cause of an acute non-compressive transverse myelopathy is not apparent.

\section{REFERENCES}

Brinar, V. V., Habek, M., Zadro, I., Barun, B., Ozretic, D., and Vranjes, D. (2008). Current concepts in the diagnosis of transverse myelopathies. Clin. Neurol. Nurosurg. 110, 919-927.

Jacob, A., and Weinshenker, B. G. (2008). An approach to the diagnosis of acute transverse myelitis. Semin. Neurol. 28, 105-120.

Jacobi, C., Stingele, K., Kretz, R., Hartman, M., StorchHagenlocher, B., Breitbart, A., and Wildeman, B. (2006). Neuromyelitis optica (Devic's syndrome) as first manifestation of systemic lupus erythematosus. Lupus 15, 107-109.

Polman, C. H., Reingold, S. C., Edan, G., Filippi, M., Hartung, H. P., Kappos, L., Lublin, F. D., Metz, L.
M., McFarland, H. F., O'Connor, P. W., SandbergWollheim, M., Thompson, A. J., Weinshenker, B. G., and Wolinsky, J. S. (2005). Diagnostic criteria for multiple sclerosis: 2005 revisions to the "McDonald Criteria”. Ann. Neurol. 58, 840-846.

Received: 18 March 2010; accepted: 19 March 2010; published online: 23 April 2010.

Citation: Chakravarty A (2010) The challenge of acute noncompressive transverse myelopathies. Front. Neur. 1:6. doi: 10.3389/fneur.2010.00006

This article was submitted to Frontiers in Spinal Cord Medicine, a specialty of Frontiers in Neurology.

Copyright () 2010 Chakravarty. This is an open-access article subject to an exclusive license agreement between the authors and the Frontiers Research Foundation, which permits unrestricted use, distribution, and reproduction in any medium, provided the original authors and source are credited. 